\title{
Revus
}

Journal for Constitutional Theory and Philosophy of Law / Revija za

ustavno teorijo in filozofijo prava

39 | 2019

Revus (2019) 39

\section{En defensa de una concepción constructivista de la interpretación jurídica}

In defence of a constructivist conception of legal interpretation

Isabel Lifante Vidal

\section{(2) OpenEdition}

Journals

Edición electrónica

URL: http://journals.openedition.org/revus/5423

DOI: $10.4000 /$ revus.5423

ISSN: 1855-7112

Editor

Klub Revus

Referencia electrónica

Isabel Lifante Vidal, "En defensa de una concepción constructivista de la interpretación jurídica »,

Revus [Online], 39 | 2019, Online since 13 December 2019, connection on 01 January 2020. URL :

http://journals.openedition.org/revus/5423; DOI : 10.4000/revus.5423

Este documento fue generado automáticamente el 2 enero 2020.

All rights reserved 


\title{
En defensa de una concepción constructivista de la interpretación jurídica
}

\author{
In defence of a constructivist conception of legal interpretation
}

Isabel Lifante Vidal

\section{Algunas cuestiones conceptuales para comenzar}

1 Suele sostenerse que la interpretación en general consiste en atribuir o precisar el sentido de algo y que, en el ámbito jurídico, esta actividad resulta de suma importancia dadas las múltiples indeterminaciones que pueden afectar al Derecho. Ahora bien, en cuanto pretendemos caracterizar con algún detalle en qué consiste esta actividad interpretativa, las discrepancias comienzan a multiplicarse y no es fácil encontrar una definición unánime de la expresión "interpretación jurídica". Por ello considero necesario empezar con algunas aclaraciones conceptuales. En algunos trabajos anteriores me he ocupado de analizar las diversas ambigüedades que encontramos al hablar de "interpretación" en general y de "interpretación jurídica en particular" todas ellas me interesa ahora rescatar dos que me parecen relevantes para exponer con claridad la concepción de la interpretación que pretendo defender. La primera ambigüedad tiene que ver con el tipo de actividad al que nos referimos al hablar de interpretación (interpretación en sentido "noético" frente a interpretación en sentido "dianoético"), mientras que la segunda está relacionada con cuál consideramos que es el objeto de la interpretación jurídica (interpretatio legis, o más en general de disposiciones jurídicas, frente a interpretatio iuris).

\subsection{Intepretación noética frente a interpretación dianoética}

2 Cuando hablamos de "interpretación" podemos referirnos o bien a una actividad o bien a su resultado; se trata de una aplicación de la extendida ambigüedad proceso- 
producto: tendríamos, así, un primer sentido de "interpretación", la interpretaciónactividad, que haría referencia al proceso o a la actividad interpretativa; y un segundo sentido, la interpretación-resultado, que se referiría al resultado o producto al que nos conduce dicha actividad. Ahora bien, lo que aquí me interesa destacar es que dentro de la interpretación como actividad podemos a su vez distinguir dos sentidos de actividad interpretativa que, siguiendo a Gianformaggio, podríamos denominar "interpretación noética" e "interpretación dianoética". Para esta autora nos encontraríamos con una interpretación como actividad noética cuando se produce una captación del significado como un pensamiento intuitivo, es decir, una captación intelectual inmediata de una realidad inteligible; mientras que hablaríamos de interpretación como actividad dianoética cuando se requiera un pensamiento discursivo, una argumentación ${ }^{2}$. Lo que subyace a estos dos sentidos de interpretación sería la misma distinción que han desarrollado recientemente psicólogos como Daniel Kahneman (2011) entre dos sistemas de pensamiento: uno rápido o intuitivo y otro lento o reflexivo que se vincularía con la deliberación.

3 Es fácil percatarse de que el primer sentido, el de la interpretación noética, hace referencia a una actividad omnipresente: en cualquier situación de comunicación necesitamos captar el significado, mientras que el segundo, el de la interpretación dianoética, hace referencia a una actividad que solo será necesaria en aquellas situaciones en las que se planteen dudas sobre el significado a atribuir al objeto interpretado. Es en la caracterización de este segundo sentido de actividad interpretativa en el que me quiero centrar en este trabajo, aunque por supuesto hemos de ser conscientes de que no se trata de actividades "excluyentes": cualquier actividad interpretativa dianoética comenzará con una captación de significado (aunque éste sea impreciso). Por decirlo de otro modo, la interpretación noética estará presente tanto en todos los casos, tanto en los claros como en los dudosos; mientras que la interpretación dianoética sólo será necesaria en los que aparezcan dudas.

\subsection{Interpretatio legis frente a interpretatio iuris}

4 Para presentar esta segunda ambigüedad tenemos que comenzar haciendo referencia a los diversos sentidos que adopta el término interpretación en función de cuál se considera que puede ser el objeto de la interpretación. Siguiendo en este punto a Wróblewski, podemos distinguir la interpretación sensu largissimo de la interpretación sensu largo. La interpretación sensu largissimo se predica de cualquier entidad capaz de ser portadora de un sentido (es decir, cualquier objeto no natural); así, por ejemplo, se puede hablar de la "interpretación" de una acción, de una práctica social, de un acontecimiento histórico o de un cuadro; aquí por "interpretación” entenderíamos la comprensión de "cualquier objeto en tanto que fenómeno cultural". En otras ocasiones, sin embargo, utilizamos el término "interpretación" en un sentido más restringido, que tiene como objeto únicamente a las entidades lingüísticas; sería lo que Wróblewski llama interpretación sensu largo ${ }^{3}$.

5 En el concreto ámbito de la interpretación jurídica nos encontramos con una distinción clásica, vinculada también a cuál se considera como objeto de interpretación y que como vamos a ver presenta claros paralelismos con la distinción de Wróblewski. Me refiero a la distinción entre la interpretatio legis (interpretación de las disposiciones contenidas en las leyes $u$ otros documentos jurídicos autoritativos) y la interpretatio iuris 
(interpretación del Derecho en su conjunto). Tarello (1980) conectaba estas dos categorías con la evolución histórica del sentido del término "interpretación" en el ámbito jurídico ${ }^{4}$, dando a entender que el sentido moderno de interpretación jurídica se restringe a interpretación de disposiciones, pero yo no estoy muy segura de que eso sea cierto y creo que en ocasiones los juristas seguimos usando la expresión interpretación jurídica para hacer referencia a la interpretación del Derecho (como cuando, por ejemplo, se considera que problemas como las lagunas o las antinomias generan la necesidad de llevar a cabo la interpretación jurídica). Por lo que se refiere al ámbito de las teorías, nos encontramos con algunas (fundamentalmente las de orientación analítica) que manejan un concepto más estricto de interpretación referido exclusivamente a entidades lingüísticas, y cuyo objeto en el ámbito jurídico serían las disposiciones contenidas en las distintas fuentes autoritativas del Derecho. Para ellas, la actividad interpretativa estaría siempre vinculada a la resolución de una imprecisión vinculada a una expresión lingüística. Y, por otro lado, habría concepciones de la interpretación jurídica que hacen referencia a la "interpretación del Derecho" o interpretatio iuris, y que incorporarían un ámbito de actividades más amplio que el supuesto anterior: no sólo precisión del significado de expresiones lingüísticas contenidas en las disposiciones jurídicas, sino también resolución de antinomias, ponderación de principios para la resolución de lagunas, etc. El objetivo de la actividad interpretativa así entendida radicaría en reconstruir los materiales jurídicos, con la finalidad de buscar en el Derecho una solución unitaria para un problema jurídico; de este modo, bajo el rótulo de "interpretación jurídica" estas concepciones incluirían todas las actividades que se suelen agrupar bajo el nombre de "método", "integración" o "construcción jurídica" 5. Podríamos situar aquí tanto a la concepción de interpretación sostenida por Dworkin, como también a la de los realistas americanos.

6 Hay que ser conscientes de que la interpretación del Derecho implicará llevar a cabo actividades incluidas en la interpretación de la ley $\mathrm{u}$ otros materiales jurídicos autoritativos: el punto de partida del trabajo reconstructivo del Derecho han de ser las leyes, o más en general, las disposiciones jurídicas dotadas de autoridad. Pero lo que me parece más relevante destacar es la relación inversa (no tan obvia): en el ámbito jurídico la actividad de interpretar una disposición cuyo significado plantea problemas implica determinar cuál es su significado a la luz del ordenamiento jurídico en su conjunto; las decisiones adoptadas por las diferentes instancias jurídicas autoritativas (y también las de la dogmática) deben presentarse como justificadas jurídicamente, lo que supone que sean acordes con "el Derecho". Para ello habrá que llevar a cabo actividades incluidas en el sentido de "interpretación" más amplio conectado con la reconstrucción del Derecho (ponderaciones, reconstrucciones dogmáticas, sistematizaciones, etc.). Las actividades implicadas bajo ambos rótulos se encuentran por tanto interrelacionadas.

\section{El papel de los argumentos interpretativos: escepticismo, cognoscitivismo y constructivismo}

7 La actividad interpretativa (en sentido dianoético) consiste en argumentar a favor de atribuir un determinado sentido a un objeto. Pero ¿cuáles son los argumentos que se consideran pertinentes concretamente en el ámbito jurídico? Y aquí creo que encontramos una cierta unanimidad como punto de partida. Entre los trabajos 
dedicados al análisis de los catálogos de argumentos interpretativos, el desarrollado por el Comparative Legal Research Group coordinado por MacCormick y Summers (1991) merece especial atención. En él se comparan los tipos de argumentos interpretativos que se utilizan -aunque en ocasiones bajo distintos nombres- en los sistemas jurídicos analizados, que son los de nuestra cultura jurídica (sistemas tanto de Common Law como de Derecho continental) para la interpretación de las leyes ${ }^{6}$. En primer lugar, están los argumentos interpretativos que apelan al lenguaje mismo (ordinario o técnico) como fuente de razones para favorecer una u otra interpretación (los argumentos "lingüísticos"). En segundo lugar, nos encontramos con los argumentos "sistemáticos", que serían aquellos que se orientan hacia una comprensión aceptable de un texto jurídico visto como parte de un todo (el sistema). En esta categoría se incluirían seis tipos de argumentos (que apoyarían a las interpretaciones que mejor armonizaran (1) con otras interpretaciones de términos o expresiones ya usadas en otras partes del mismo texto legal, o (2) en precedentes judiciales, o (3) que armonizaran con provisiones análogas contenidas en otras leyes, o (4) con construcciones dogmáticas, o (5) con los principios generales del derecho aplicables al caso, o (6) con el entendimiento históricamente desarrollado sobre su objeto y propósito o sobre su corrección). En tercer lugar, estarían los argumentos "teleológicos y deontológicos", que son aquellos que ofrecen las interpretaciones que mejor se acomodan al propósito o fin de la norma o que ofrecen la mejor realización de un valor considerado fundamental por el sistema jurídico. Por último, existe la posibilidad de apelar a la intención del autor como material para elucidar el significado del texto autoritativo; pero MacCormick (2010) señala que la intención puede estar ligada estrechamente a elementos lingüísticos de semántica o sintaxis, a todos los elementos del contexto sistémico de un texto jurídico, o a los objetivos perseguidos o principios respaldados por el legislador bien como un cuerpo histórico o como un legislador idealmente racional, por lo que considera que debe ser tratado como un argumento "transcategórico"?.

8 Ahora bien, la cuestión relevante que debemos plantearnos es por qué son precisamente esos los argumentos con que contamos para atribuir significado en el Derecho y a cuál de ellos debe darse prioridad en caso de que avalen interpretaciones incompatibles ${ }^{8}$. En este sentido podemos encontrar distintas concepciones interpretativas, dependiendo precisamente de cómo responden a estas cuestiones.

Tenemos un primer grupo de teorías que se limitan a constatar la existencia de los diferentes argumentos interpretativos, señalando que en caso de conflicto entre ellos no habría nada más que decir. Se trataría de teorías que suelen calificarse como "teorías escépticas" de la interpretación, según las cuales la naturaleza de la actividad interpretativa sería la de un acto de mera voluntad y no existirían criterios de corrección que guíen la elección entre las distintas posibilidades interpretativas, del mismo modo que para ellas tampoco tendría sentido preguntarse por la justificación de los distintos cánones: son los que son simplemente porque de hecho se usan (por lo que es de suponer que, para estas teorías, los mismos podrían cambiar, pues no hay nada que los justifique ni los haga necesarios). En el extremo opuesto nos encontramos con las llamadas "teorías cognoscitivistas" que consideran que la naturaleza de la actividad interpretativa es la de ser un acto de conocimiento: existen por tanto interpretaciones verdaderas, del mismo modo que existen interpretaciones falsas. Dentro de estas teorías podemos distinguir a su vez entre aquéllas que consideran que el criterio de 
verdad viene dado por el significado literal de las palabras usadas por el legislador (las llamadas teorías literalistas de la interpretación ${ }^{9}$ ) y aquellas que consideran que viene dado por su correspondencia con las intenciones del legislador (las llamadas teorías subjetivistas o intencionalistas ${ }^{10}$ ). En ambos casos se trata de teorías que darían una prioridad absoluta respectivamente a los argumentos semánticos o a los genéticos, los cuales acuden a una argumentación fundamentalmente empírica: para la teoría del significado literal, una interpretación sería correcta cuando coincida con el significado que de hecho se da a las palabras (suponiendo la univocidad del mismo), mientras que para la teoría intencionalista, una interpretación sería correcta cuando coincida con la intención que de hecho tenía el legislador (suponiendo la existencia y posibilidad de conocimiento de dicha intención).

10 Entre esos dos extremos (escepticismo y cognoscitivismo) suele situarse otro grupo de teorías a las que en ocasiones se las llama "teorías intermedias", cuyo ejemplo paradigmático sería la teoría de Hart ${ }^{11}$. Pero, ¿es realmente posible esta vía intermedia? Guastini, por ejemplo, lo niega, considerando que las dos alternativas anteriores son excluyentes entre sí y que, en realidad, la propuesta de Hart no supondría una tercera opción, puesto que presentaría a la actividad interpretativa en unos casos como actividad puramente cognoscitiva (en los casos fáciles) y en otros como actividad puramente volitiva (en los casos difíciles) ${ }^{12}$. Y, además, dado que lo que estamos intentando caracterizar sería lo que hemos denominado interpretación dianoética, debemos considerar que nuestros supuestos son todos casos difíciles, en los que se plantea una duda sobre el significado (este planteamiento es el que lleva a Guastini a considerar que la única concepción aceptable para la interpretación sería una teoría escéptica).

11 Pero creo que existe otra posible lectura de en qué puede consistir una "teoría intermedia" mucho más plausible. La actividad interpretativa (en los casos difíciles) sería una actividad de naturaleza argumentativa, que pretende reconstruir de la mejor manera posible el significado a atribuir al Derecho. El resultado de la actividad interpretativa se suele formular como un enunciado del tipo: "D significa N". Pero es importante señalar que ese enunciado es la conclusión de una argumentación en la que se dan razones para preferir el significado "N" a otros posibles significados. Ese enunciado (cuando es usado en un discurso interpretativo, y no en un discurso descriptivo de las interpretaciones realizadas por otro ${ }^{13}$ ) vendría a ser equivalente a un enunciado del tipo "D debe ser entendido como N", o "Es mejor entender D en el sentido $\mathrm{N}$ que en cualquier otro sentido". Es decir, los enunciados interpretativos incorporan una pretensión de corrección, aunque dicha pretensión no tiene por qué definirse en términos de verificabilidad empírica, sino que -como vamos a ver- incorporan evaluaciones.

12 Desde esta perspectiva, la interpretación jurídica no sería, por tanto, ni un mero acto de conocimiento (como pretenden los cognoscitivistas) ni tampoco un mero acto de voluntad (como afirman los escépticos), aunque puede contener algo de ambas actividades en las diversas etapas o momentos del proceso interpretativo (se inicia con actos cognoscitivos y finalizará con la adopción de una decisión). La actividad interpretativa sería una actividad argumentativa que se inserta en el ámbito más amplio del razonamiento práctico general. En este sentido, la teoría de la interpretación jurídica que yo defiendo puede ser considerada una parte de la teoría de la argumentación jurídica y, como tal, no puede pretender ser exclusivamente 
descriptiva o instrumental ${ }^{14}$. Debe servir también como guía para la práctica jurídica. Para este objetivo debe incorporar elementos normativos o evaluativos. De este modo, la teoría de la interpretación jurídica que estoy intentando elaborar aquí tiene un alcance reconstructivo: intenta describir las prácticas jurídicas existentes, pero también presentar aquellos aspectos de la misma que usamos justificadamente para evaluarlas.

La cuestión relevante sería, por tanto, determinar qué tipo de criterios de evaluación son los que operan en el caso de la actividad argumentativa en el concreto ámbito de la interpretación jurídica. Como alternativa a los criterios basados en la verificabilidad empírica a los que hemos visto que acudían las teorías cognoscitivistas, nos encontramos con otras opciones, tales como la aceptabilidad pragmática a la luz de los fines y valores que pretende desarrollar la práctica en cuestión. Se trataría aquí de lo que ha dado en llamarse una "teoría constructivista o dependiente de valores". Como vamos a ver, para una teoría de este tipo la interpretación es una actividad valorativa que lleva a cabo un proceso reconstructivo de los materiales jurídicos en el cual se establezcan los valores y objetivos perseguidos por el Derecho -de manera que éste se presente como un todo coherentemente orientado a la persecución de fines considerados moralmente valiosos- y se determine qué concreta interpretación los desarrolla en mayor medida.

El siguiente cuadro pretende esquematizar las diferentes concepciones interpretativas a las que me he referido anteriormente:

\begin{tabular}{|l|l|l|}
\hline & Naturaleza de la actividad Interpretativa & Criterios de corrección \\
\hline Escepticismo & Volitiva & No se aplican \\
\hline Cognoscitivismo & Cognitiva & Verificabilidad empírica \\
\hline Constructivismo & Argumentativa & Realización valor jurídico \\
\hline
\end{tabular}

Pero en realidad creo que todos los intentos de defender concepciones de la interpretación que responden a la pregunta acerca de cómo se debe interpretar el Derecho (dejando por tanto fuera el escepticismo) acaban en realidad utilizando un modelo constructivista o valorativo de la interpretación. Las diferencias entre unas y otras concepciones normativas (o prescriptivas) las vamos a encontrar en la determinación de cuál es el valor que debemos atribuir al Derecho: un valor más "formal", tal como el ideal del imperio de la ley, que nos conduce a primar el aspecto autoritativo del Derecho; o un valor más sustantivo, que nos conducirá a primar los objetivos valiosos que el Derecho pretenda desarrollar (los ideales de justicia encarnados en una combinación de los valores de igualdad y libertad).

En este sentido, MacCormick (2010) señala, por ejemplo, que detrás de cada una de las categorías de argumentos antes analizados subyace una serie de valores y principios. Así, tras la interpretación lingüística nos encontraríamos con el objetivo de preservar la claridad y la exactitud del lenguaje legislativo, así como la prohibición de la reescritura judicial retrospectiva de las palabras del legislador; tras la interpretación sistemática estaría el principio de racionalidad fundado en el valor de la coherencia e integridad del sistema jurídico; y tras la interpretación teleológica/deontológica estaría el respeto 
por la exigencia de la razón práctica de que la acción humana esté guiada por valores a alcanzar o por principios a observar. Ahora bien, si tenemos en cuenta que una importante razón justificativa para el mantenimiento de las instituciones jurídicas es precisamente la disminución del alcance de las disputas interpersonales sobre cuáles son los valores y principios que rigen en el ámbito social, este objetivo sería lo que justifica dar un peso considerable a los argumentos lingüísticos y sistemáticos en el Derecho. De modo que podemos considerar que, aunque el significado literal o la intención del legislador juegan un papel muy relevante en la interpretación jurídica, ellos serían el punto de partida, y en algunas ocasiones también pueden ser el de llegada, el proceso interpretativo implica siempre atender también a razones de por qué interpretar en un cierto sentido, razones que no pueden ser el mero dato objetivo de un significado literal o de una determinada intención.

Y, en un sentido parecido, Nino (1994) también pone en duda la clásica contraposición subjetivismo/objetivismo. Este autor señala que, pese a los sólidos argumentos que el intencionalismo (subjetivista) parece tener a su favor (y que remitirían a la idea de que el lenguaje es un medio de comunicación, y que la comunicación exige que la intención del emisor sea captada por el receptor), en realidad las cosas no son tan fáciles. En primer lugar, Nino nos recuerda que no podemos reconocer intenciones al margen de las convenciones: son las convenciones sociales lingüísticas -objetivables- las que permiten asociar determinadas palabras con cierta intención; y al mismo tiempo la intención tampoco permite asignar a las palabras significados diferentes de los que surgen de las convenciones. Pero hay además -señala Nino- varios problemas añadidos a la determinación de la intención del autor del texto jurídico que debemos tener en cuenta: la selección de los hechos que constituyen manifestaciones de la intención; la construcción de una intención a partir de la intervención de una pluralidad de órganos o de órganos colectivos; la admisibilidad de la reconstrucción de la intención atendiendo a circunstancias contrafácticas; la determinación del nivel de abstracción con el que debe describirse la intención del legislador; la reconstrucción de la intención en caso de que algunas de las creencias del legislador fueran falsas (creencias que pueden ser incluso de tipo valorativo). La conclusión a la que Nino llega después de analizar todos estos problemas es que la intención del legislador (así como la del constituyente) es siempre una construcción a partir de un cúmulo de valoraciones. Por ello, señala que le parecería más honesto aplicar directamente esas valoraciones para la selección del significado del texto a interpretar sin esa intermediación, pues la misma cumpliría funciones ideológicas (ocultación de las valoraciones bajo una supuesta búsqueda "empírica" de la intención como un hecho).

\section{En defensa de una concepción constructivista de la interpretación jurídica}

\subsection{La concepción del Derecho y de la racionalidad práctica}

Mi punto de partida es que la interpretación jurídica (la dianoética) es una actividad de naturaleza argumentativa inserta en el ámbito más amplio del razonamiento práctico general. De este modo, las respuestas a la pregunta sobre cómo debe interpretarse el Derecho se encuentran en gran medida predeterminadas por los presupuestos teóricos 
adoptados respecto a dos cuestiones previas: la naturaleza del Derecho y de la racionalidad práctica en general.

Respecto a la primera cuestión, hay a su vez dos variables a tomar en cuenta ${ }^{15}$. Por un lado, la relativa a la idea de Derecho que se sostiene; en este sentido, nos encontramos unas concepciones para las cuales el Derecho sería un conjunto de normas (lo que Dworkin denomina concepto taxonómico de Derecho ${ }^{16}$ ) y otras concepciones para las cuales se trata de una práctica social (una actividad o una empresa) orientada a la persecución de ciertos fines. Las primeras concepciones priman el aspecto estructural y organizativo del fenómeno jurídico, mientras que las segundas dan mayor relevancia a los aspectos teleológicos y valorativos del mismo. Y, por otro lado, nos encontramos con la variable relativa a si estas concepciones se adhieren o no a la tesis positivista de la separación conceptual entre el Derecho y la moral. Pese a lo que pudiera considerarse a primera vista, estas dos variables son relativamente independientes, de modo que sería posible sostener una idea del Derecho como conjunto de normas y defender al mismo tiempo una conexión conceptual entre el Derecho y la moral ${ }^{17} \mathrm{y}$, a la inversa, sostener una idea del Derecho como práctica social, pero desde una perspectiva positivista (pensemos, por ejemplo, en el segundo Ihering).

Yo me adhiero a una idea de Derecho como una práctica social y a una concepción postpositivista (o no positivista) del Derecho. Por un lado, una práctica social, como yo la entiendo ${ }^{18}$, no está sólo, ni siquiera principalmente, constituida por una serie de conductas regulares empíricamente observables. Cualquier práctica implica una serie de valores que deben intentar realizarse a través de la práctica. Estos valores constituyen el aspecto normativo de la práctica y sirven como criterios para evaluar la elección de una posible solución sobre otras a los problemas planteados en la práctica. Y, por otro lado, una concepción postpositivsta del Derecho sostiene que cuando nos aproximamos al Derecho desde ciertas perspectivas (por ejemplo si lo que nos interesa es un concepto "aspiracional" o "doctrinal" de Derecho) no puede mantenerse la separación conceptual entre el Derecho y la moral, aunque ello no sea obstáculo para que, desde otras perspectivas, dicha distinción sea totalmente operativa. El Derecho de un Estado constitucional no puede verse exclusivamente como un instrumento para lograr cualesquiera objetivos sociales, sino que incorpora valores morales ${ }^{19}$.

21 Respecto a la concepción de la racionalidad práctica, hay dos posibles posturas a adoptar: la primera negaría la existencia de la racionalidad en el ámbito práctico, de modo que la argumentación en el ámbito de la razón práctica (a diferencia de lo que ocurriría con el ámbito de la ciencia) no podría ser calificada como una actividad "racional", pues no habría criterios objetivos para su evaluación; la segunda postura aceptaría la racionalidad en dicho ámbito, y por tanto la existencia de criterios que permiten evaluar la corrección de las argumentaciones prácticas.

22 Si alguien es irracionalista o escéptico en el ámbito de la racionalidad práctica, entonces obviamente para él la pregunta a propósito de cómo se debe interpretar algo (en nuestro caso el Derecho) carecería de sentido desde un punto de vista objetivo. La interpretación sería concebida como un acto de naturaleza volitiva ajeno a cualquier evaluación racional. Desde esta perspectiva, la única actividad "racional" que se puede llevar a cabo sería una actividad meramente descriptiva de las prácticas interpretativas existentes, pero preguntarse por los criterios de evaluación de las mismas excedería los límites de lo que una "teoría de la interpretación" puede albergar. Un teórico escéptico puede dar cuenta de cuáles son los argumentos interpretativos que de hecho se usan en 
una comunidad, pero su investigación deberá detenerse aquí, no pudiendo justificar por qué son esos argumentos y no otros, ni siquiera dar cuenta de por qué en todas las comunidades se usan los mismos tipos de argumentos interpretativos (lingüísticos, sistemáticos y teleológico-deontológicos ${ }^{20}$; ni tampoco determinar cuál de ellos debería tener más peso en un determinado caso en el que entren en conflicto entre sí.

Pero esta tesis plantea además una pequeña paradoja: Si el teórico de la interpretación jurídica debe limitarse a dar cuenta de las prácticas interpretativas realmente existentes, y dado que dichas prácticas incorporan la pretensión de corrección, el teórico deberá dar cuenta de dicha pretensión. Los enunciados interpretativos jurídicos incorporan una elección entre varias posibles alternativas y son el resultado de una argumentación. No tiene sentido argumentar, dar razones a favor de algo, si no presuponemos que hay buenas y malas razones o razones mejores que otras, es decir, criterios que nos permitan discriminar entre ellas, aunque puede discutirse cuáles son dichos criterios o cómo se aplican a un caso concreto, pero lo que no se discute es la propia existencia de esos criterios. La actividad argumentativa presupone en este sentido aceptar la racionalidad en aquel ámbito en el que nos encontremos. Si adoptamos -como creo que debe hacerse- una concepción de la actividad interpretativa como una actividad argumentativa "racional", ello nos lleva a considerar que, por un lado, se trata de una actividad orientada a un fin, es decir, que sirve a un propósito; y, por otro lado, que existen criterios para evaluar una argumentación, considerándola como buena o mala, o mejor o peor que otra ${ }^{21}$. Para contestar a la pregunta a propósito de cómo interpretar el Derecho, deberemos entonces determinar en primer lugar cuál es el propósito de tal actividad (para qué interpretamos el Derecho) y en segundo lugar determinar en qué consisten los criterios que nos permitan evaluar dichas interpretaciones (y que estarán en función de la respuesta dada a la primera pregunta). Todas las concepciones no escépticas a propósito de la interpretación jurídica deben por tanto ofrecer respuesta a estas dos cuestiones: ¿para qué interpretamos?, y ¿cómo debemos interpretar, o cuáles son los criterios que utilizamos para evaluar las interpretaciones?

\section{2 ¿Para qué interpretamos el Derecho?}

La primera cuestión será entonces ¿para qué interpretar el Derecho?, ¿cuál es el fin o propósito de la actividad interpretativa en el ámbito jurídico? Pues bien, debemos ser conscientes de que la interpretación "jurídica" no hace referencia a cualquier posible actividad de atribución de significado a textos jurídicos (leyes, reglamentos, sentencias, actos administrativos... $)^{22}$. Creo que la actividad interpretativa jurídica se define no sólo por el hecho de que su objeto sean textos jurídicos, sino también por el propósito con el que se lleva a cabo dicha actividad y que no es otra que la de contribuir -en distinta medida- a la conformación de la práctica jurídica ${ }^{23}$. Dicho de otro modo, cuando caracterizamos a una interpretación como "jurídica" necesitamos incluir la referencia al contexto en que se lleva a cabo dicha interpretación, y no sólo al tipo de objeto que se interpreta $^{24}$. Por supuesto que alguien (pensemos en un filólogo o un sociólogo) puede tener interés en atribuir significado a un texto jurídico en una clave distinta, desde fuera, sin compartir por tanto el objetivo de contribuir a conformar esa práctica jurídica; pero no creo que esta interpretación, aun siendo interpretación de textos jurídicos, fuera considerada como "interpretación jurídica" en sentido estricto, al menos no formaría parte de lo que entendemos que hacen los juristas (teóricos o 
prácticos) en cuanto tales, es decir, en su tarea de contribuir a determinar qué concretos derechos y obligaciones establece un determinado Derecho. La interpretación jurídica no es simplemente una interpretación de textos jurídicos, sino una interpretación en el seno de una práctica normativa vinculada a textos.

En este sentido, las teorías que distinguen tipos interpretativos jurídicos atendiendo a los sujetos que interpretan suelen destacar dos categorías ${ }^{25}$ : la interpretación judicial o, más en general, de los aplicadores del Derecho, que sería una interpretación dirigida a buscar la solución de un determinado problema práctico y la interpretación doctrinal (la llevada a cabo por los juristas teóricos), que sería fundamentalmente una interpretación en abstracto, dirigida a la reconstrucción del sistema ${ }^{26}$. Lo que tienen en común es que ambos sujetos se caracterizarían por adoptar (aunque con distinta intensidad) el punto de vista del aceptante del Derecho y, en este sentido, estarían comprometidos con los fines y valores que el Derecho pretende desarrollar. El intérprete del Derecho sería por tanto un participante en la práctica jurídica que, como tal, ayuda a su conformación.

Es cierto que las concepciones escépticas de la interpretación (como las de Kelsen o Guastini ${ }^{27}$ ) estarían en desacuerdo con esta caracterización. Para ellos la interpretación llevada a cabo por la ciencia del Derecho, sería una actividad "cognitiva" que tendría como propósito conocer las posibles interpretaciones atribuibles a un texto jurídico sin ningún compromiso práctico. Su objetivo sería por tanto puramente teórico meramente cognoscitivo- y no práctico: ni pretenden contribuir a conformar la práctica ni mucho menos a mejorarla. Ahora bien, dudo que haya muchos juristas que efectivamente lleven a cabo actividades interpretativas dirigidas simplemente a mostrar los posibles significados atribuibles a una disposición; pero incluso aunque los hubiera podemos pensar que estas actividades solo tienen sentido ("sirven para algo") si son concebidas como instrumentalmente útiles para una actividad posterior de carácter práctico (la propuesta de una reforma legislativa, la defensa de una determinada solución a un problema jurídico...). En este sentido, la interpretación "cognitiva" de la que habla Guastini (o la "científica" en terminología kelseniana) podría ser considerada como una etapa de un proceso interpretativo más amplio que incluya en último término una aplicación práctica. De este modo, se reivindica el carácter práctico de la teoría y de la "ciencia" del Derecho, las cuales no pueden reducirse a discursos meramente descriptivos.

La actividad interpretativa es por tanto una actividad conformadora de la práctica jurídica. El intérprete jurídico es un participante en dicha práctica y no un mero observador de la misma. El autor de aquello que se interpreta (las autoridades jurídicas creadoras del Derecho) y el intérprete participarían en un mismo proyecto o empresa (el Derecho) y en este sentido compartirían el propósito general perseguido por el proyecto y ambos tendrían el deber de intentar avanzar en dicho proyecto, mejorándolo en la medida de sus posibilidades ${ }^{28}$. ¿Qué debe buscar entonces el intérprete del Derecho al desempeñar su rol ${ }^{29}$ ? ¿Cuál es el objetivo que debe guiar esta actividad interpretativa? Obviamente el mismo objetivo que ha de perseguir cualquier otro participante en dicha práctica: su mejora.

Claro que puede pensarse que este compromiso con la conservación y mejora de la práctica sólo sería exigible para aquellos intérpretes a los que su rol en el Derecho les exige independencia e imparcialidad (el caso prototípico sería el de los jueces) pero no para otros participantes en la práctica; pienso fundamentalmente en los abogados, que 
operan en defensa de intereses particulares que pueden no coincidir con -aunque deben tener cabida en- la defensa de los propósitos atribuibles al Derecho. Ello es cierto, pero creo que no implica que estas interpretaciones no deban estar también, aunque en algún sentido más indirecto -podríamos decir: con muchas más mediaciones-, dirigidas a mejorar la práctica en su conjunto ${ }^{30}$. Ahora bien, no debemos olvidar que, en la medida en que estos otros intérpretes pretendan que sus propuestas interpretativas sean asumidas por terceros y en particular por los encargados de hacer efectivo el Derecho (por las diversas autoridades jurídicas), habrán de adoptar esa misma perspectiva del aceptante imparcial, y habrán de presentar las razones que avalen su opción interpretativa en términos de los propósitos y valores de la propia práctica jurídica.

\section{3 ¿Cómo debemos interpretar el Derecho?}

30 Si aceptamos lo anterior (la idea de que el objetivo de la interpretación jurídica ha de ser la mejora de la práctica), la pregunta a propósito de cómo interpretar el Derecho nos remite a una cuestión previa: qué es lo que hace valioso al propio Derecho. El Derecho se interpreta para atribuirle un sentido en aquellos casos en que, prima facie, admite más de una posible lectura y la comparación entre estas distintas interpretaciones prima facie posibles que compiten entre sí ha de hacerse atendiendo a los propios valores que el Derecho pretende realizar.

Los criterios de corrección en al ámbito de la actividad interpretativa nos remiten de este modo a la cuestión a propósito del valor del Derecho: el ideal de Estado de Derecho. Se trata de un concepto controvertido para el que encontramos diversas concepciones que compiten entre sí y que pueden ser más o menos formalistas, dependiendo del peso que se dé a cada una de las dos dimensiones que una teoría del Derecho debe integrar: la dimensión autoritativa y la dimensión axiológica; o equidad procedimental y justicia sustantiva.

En el contexto anglosajón es usual contraponer un concepto de "rule of law" restringido (a veces calificado como "formal" o "legal") ${ }^{31}$ a otro más sustantivo. Ese concepto restringido sería el más extendido y sería el que maneja -entre otros- Raz, para quien:

[t]he rule of law is a political ideal which a legal system may lack or may possess to a greater or lesser degree. That much in common ground. It is also to be insisted that the rule of law is just one of the virtues which a legal system may possess and by which it is to be judged. It is not to be confused with democracy, justice, equality (before the law or otherwise), human rights of any kind or respect for persons or for the dignity of man (Raz 1979: 211).

Sin embargo, en la literatura española solemos usar el concepto de "Estado de Derecho" (que es tomado de la tradición alemana como traducción del Rechtsstaat). Siguiendo las ya clásicas reflexiones de Elías Díaz, podemos considerar que el concepto de "Estado de Derecho" opera como un rótulo evaluativo para designar a aquellos Estados que cumplen (al menos en determinado grado) con ciertas exigencias normativas que implican el sometimiento del Estado a su propio Derecho, a través de la regulación y el control de todos los poderes y actuaciones del Estado por medio de leyes; leyes que deben haber sido creadas según procedimientos de libre y abierta participación popular. Junto al requisito del imperio de la ley (entendiendo por "ley", aquella que ha sido democráticamente producida), que constituiría el primer ingrediente -con prioridad lógica e histórica frente al resto- de la noción Estado de Derecho, se incluirían 
también los siguientes rasgos definitorios: “b) División de poderes: legislativo, ejecutivo y judicial. c) Legalidad de la Administración: actuación según ley y suficiente control judicial. d) Derechos y libertades fundamentales: garantía jurídico formal y efectiva realización material" (Díaz 1992: 31).

Pero estos distintos componentes del ideal no se encuentran en el mismo nivel: mientras que el primero (el imperio de la ley) goza de prioridad tanto lógica como histórica frente al resto, es precisamente el objetivo de hacer efectivos los derechos humanos como exigencias morales lo que dota de valor a las instituciones que lo hacen posible, y que conformarían el modelo jurídico-político que denominamos Estado de Derecho. De modo que el último elemento (la garantía de los derechos y libertades) tendría -podríamos decir- una prioridad justificativa frente al resto.

Esa misma idea de la instrumentalidad del imperio de la ley ha sido desarrollada por Laporta (2007), para quien se trata de un ideal regulativo: un conjunto de exigencias éticas respecto al poder y al Derecho que deben ser satisfechas en la mayor medida posible ${ }^{32}$. El valor moral de esta exigencia la encuentra este autor en su vinculación directa con la autonomía personal. La existencia de normas que nos proporcionan un cierto grado de previsibilidad de las relaciones sociales es una condición necesaria (aunque claramente no suficiente) para el desarrollo de la autonomía personal, entendida como la exigencia de que la vida del ser humano sea algo definido por él mismo en un marco de libertad personal y de racionalidad proyectiva. A partir de esta relación, algunos autores (como el propio Laporta) consideran que hay que optar por una comprensión "formalista" del valor del Derecho. La seguridad o certeza jurídica (conectada a la maximización de la previsibilidad) se presenta como dependiente exclusivamente de ciertos rasgos formales del Derecho, en los cuatro sentidos de formalidad que señalan Atiyah y Summers ${ }^{33}$ : formalidad autoritativa (cuando tanto la validez como la jerarquía de una norma se hace depender del origen de la misma), formalidad en cuanto al contenido (que depende del grado en el que es definido mediante un fiat y no por referencia directa a las razones sustantivas que la justificarían), formalidad interpretativa (primacía de la interpretación literal frente a otros métodos interpretativos que aludan a fines o valores subyacentes) y formalidad perentoria (dificultad que presenta una razón para ser desplazada por las razones en contra). Desde esta perspectiva "formalista", la previsibilidad de un Derecho dependerá de que la aplicación de sus normas -que han de ser identificadas por su origen autoritativo- pueda realizarse de manera independiente de las razones sustantivas que las justifican, lo que implica que adopten la forma de reglas de acción y que gocen de una completa autonomía semántica, y su interpretación se limite a constatar su significado literal. La situación óptima parecería ser la aplicación del Derecho a través de un mero razonamiento deductivo. Es por esta razón (al fin y al cabo, como hemos visto, basada en una determinada concepción del valor del Derecho) por lo que algunos autores defienden una concepción literalista (o intencionalista) de la interpretación jurídica. Ahora bien, esta concepción parece olvidar que muchos de los problemas de interpretación se generan precisamente porque el significado "literal" de los textos jurídicos no es ni mucho menos unívoco y que en ocasiones dicho significado plantea dudas a la luz de otras disposiciones que han de ser tenidas en cuenta (elemento sistemático del Derecho) o de los propósitos que el establecimiento de ciertas disposiciones persiguen (elemento teleológico); o las dificultades para identificar esa intención como dato objetivo. 
Pero es que, además, esa concepción formalista del valor del ideal de Estado de Derecho se olvida de que la previsibilidad no es sólo una propiedad graduable, que puede darse en mayor o menor medida, sino también "compleja", en el sentido de que se proyecta en diversas dimensiones. Una de ellas sería la dimensión objetiva, que se ocupa de determinar qué nos permite prever el Derecho. Pero junto a ella, hay también una dimensión subjetiva que sería la que se refiere a quién puede prever ${ }^{34}$. El grado de previsibilidad dependerá aquí de cuántos individuos estén en situación de poder llevar a cabo las previsiones. Y si distinguimos -como hizo Llewellyn (1933: 58) ${ }^{35}$ - entre la seguridad de los legos y la seguridad de los juristas, habría que admitir que la primera no depende tanto de una predeterminación total del contenido de las decisiones judiciales en reglas jurídicas preexistentes. Lo que permite orientarse a la población en general y lo que, por tanto, contribuye a generar la previsibilidad necesaria para que puedan desarrollar satisfactoriamente sus planes de vida es fundamentalmente la capacidad de reconocer los principios que subyacen a las reglas y, en último término, la congruencia entre las normas jurídicas y los modos de vida. Si somos conscientes de la relevancia de los principios, entenderemos cómo también las razones sustantivas, y no sólo las formales, cumplen un papel fundamental en la generación de la previsibilidad. Por otro lado, hay que tener en cuenta que hay regulaciones que afectan típicamente a clases de sujetos con intereses enfrentados: consumidores/proveedores, empleados/ trabajadores, etc. Y en ocasiones una misma normativa puede generar grados distintos de previsibilidad para cada una de las clases de sujetos afectados por ella (pues tienen distintas posibilidades de acceso a la información relevante, pueden estar interesados en la previsibilidad de aspectos distintos), de modo que es posible que lo que incrementa la previsibilidad relevante para una parte, la disminuya para la otra.

En consecuencia, creo que la previsibilidad que consideramos valiosa y la que, por tanto, el ideal del Estado de Derecho nos obliga a maximizar será aquella que afecte a expectativas razonablemente fundadas (es decir, expectativas que han de ser consideradas legítimas a la luz de los principios y valores reconocidos por el propio Derecho). Si esto es así, debemos admitir que la seguridad jurídica, no puede ser concebida como un valor dependiente únicamente de rasgos formales del Derecho y que no es cierto tampoco que pueda desarrollarse en igual medida independientemente de la justicia o injusticia del Derecho del que se predica.

Si ahora nos fijamos en las distintas categorías de argumentos interpretativos a los que antes hemos hecho referencia, algunas de ellas se conectan directamente con la primera dimensión del Derecho: la autoritativa (los argumentos lingüísticos y también los genéticos o pragmáticos que apelan a la "intención" del legislador y quizás los sistemáticos); mientras que los argumentos teleológicos y deontológicos se conectarían directamente con la segunda dimensión: la valorativa. La interpretación ha de encajar en los materiales jurídicos (el significado a atribuir ha de estar dentro de los posibles atendiendo a las reglas semánticas, a los propósitos, a la reconstrucción sistemática de los materiales). Ir más allá sería inventar el Derecho, no interpretarlo. Aquí radica la relevancia de la dimensión autoritativa en la interpretación del Derecho. Ahora bien, cuando estos argumentos avalan varias posibilidades interpretativas, la tarea del intérprete sigue sometida a Derecho (obviamente entendiendo Derecho en un sentido no positivista, sino en el sentido antes mencionado): es la práctica jurídica la que exige que se opte por la interpretación que hace de esos materiales jurídicos autoritativos el mejor ejemplo posible de Derecho, es decir, el que lo presenta como más justo. Es por 
ello que creo que una concepción valorativa de la interpretación en el ámbito jurídico nos reenvía en último término a la necesidad de desarrollar una teoría ético-política ${ }^{36}$, produciéndose una integración de las distintas esferas de la racionalidad práctica: Derecho, moral y política.

-Agradecimientos.- Este trabajo ha contado con el apoyo del Ministerio de Economía y Competitividad a través del proyecto de investigación DER2017-86643-P. Una versión previa fue presentada y discutida en el congreso "En teoría hay mujeres (en teoría)" (Universitat Pompeu Fabra, Barcelona, 27-28 de septiembre de 2018). Quiero agradecer a todos los participantes en ese congreso y particularmente a Silvia Zorzetto por su estimulante contraponencia. También quiero agradecer a Victoria Roca, quien cuidadosamente leyó y discutió conmigo la versión inglesa de este artículo.

\section{BIBLIOGRAFÍA}

Aguiló Regla, J. (in press). En defensa del Estado constitucional de Derecho. Doxa. Cuadernos de filosofía del Derecho, 42.

Atienza, M. (2013). Curso de argumentación jurídica. Madrid, Spain: Trotta.

Atienza, M. (2017). Filosofía del Derecho y transformación social. Madrid, Spain: Trotta.

Atiyah, P.S., \& Summers, R. (1987). Form and Substance in Anglo-American Law. Oxford, England: Clarendon Press,

Barber, N. (2004). Must Legalistic Conceptions of the Rule of Law have a social dimension? Ratio Iuris, 4, 474-488.

Bobbio, N. (1938). L'analogia nella logica del Diritto. Turin, Italy: Università di Torino.

Craig, P. (1997). Formal and Substantive Conceptions of the Rule of Law: An Analytical Framework. Public Law, 3, 467-487.

Díaz, E. (1992). Estado de Derecho y sociedad democrática (8th ed.). Madrid, Spain: Taurus.

Dworkin, R. (1986). Law's Empire. Cambridge, Mass.: Belknap Press.

Dworkin, R. (2010). ¿Deben nuestros jueces ser filósofos? ¿Pueden ser filósofos? [Must our judges be philosophers? Can they be philosophers?] Isonomía. Revista de Teoría y Filosofía del Derecho, 32, 7-29.

Dworkin, R. (2014). Justicia para erizos (H. Pons, Trans.). México: Fondo de Cultura Económica.

Fuller, L.L. (1934). American Legal Realism. University of Pennsylvania Law Review, 82(5), 429-462.

Fuller, L.L. (1969). The Morality of Law. New Haven, Ct. \& London, England: Yale University Press. Gardner, J. (2012). The Supposed Formality of the Rule of Law. In Law as a Leap of Faith: Essays on Law in General (pp. 195-220). Oxford, England: Oxford University Press. 
Gianformaggio, L. (1987). Lógica y argumentación en la interpretación jurídica o tomar a los juristas intérpretes en serio [Logica e argomentazione nell'interpretazione giuridica ovvero i giuristi interpreti presi sul serio]. Doxa. Cuadernos de filosofía del Derecho, 4, 87-108.

Grabowski, A. (2015). Clara non sunt interpretanda vs. omnia sunt interpretanda. A Never-Ending Controversy in Polish Legal Theory? Revus. Journal for Constitutional Theory and Philosophy of Law, 26, 67-97.

Guastini, R. (1999). Distinguiendo. Estudios de teoría y metateoría del Derecho (J. Ferrer, Trans.). Barcelona, Spain: Gedisa.

Guastini, R. (2008). Nuovi Studi sull'interpretazione. Roma, Italy: Aracne.

Guastini, R. (2012). El escepticismo jurídico replanteado. Discusiones, 11, 27-57.

Guastini, R. (2014). Interpretar y argumentar (S. Álvarez, Trans.). Madrid, Spain: Centro de Estudios Políticos y Constitucionales,.

Hart, H.L.A. (1983). American Jurisprudence through English Eyes: The Nightmare and The Noble Dream. In Essays in Jurisprudence and Philosophy (pp. 123-144). Oxford, England: Oxford University Press.

Hart, H.L.A. (1968). Punishment and Responsibility. Oxford, England: Clarendon Press.

Kahneman, D. (2011). Thinking, fast and slow. New York, NY: Farrar, Straus and Giroux.

Kelsen, H. (1986). Teoría pura del Derecho (R. Vernengo, Trans.). México: UNAM. (Original work published 1960).

Laporta, F.J. (2007). El imperio de la ley. Una visión actual. Madrid, Spain: Trotta.

Lifante Vidal, I. (2013). Seguridad jurídica y previsibilidad. Doxa. Cuadernos de filosofía del Derecho, $36,85-105$.

Lifante Vidal, I. (2018). Argumentación e interpretación jurídica. Escepticismo, intencionalismo y constructivismo. Valencia, Spain: Tirant lo Blanch.

Llewellyn, K.N. (1933). Präjudizienrecht und Rechtsprechung in Amerika, Eine Spruchauswahl mit Besprechung. Leipzig, Germany: Weicher.

MacCormick, N. (1978). Legal Reasoning and Legal Theory. Oxford, England: Clarendon Press.

MacCormick, N. (2010). Argumentación e interpretación en el Derecho [Argumentation and interpretation in law]. Doxa. Cuadernos de filosofía del Derecho, 33, 65-78.

MacCormick, N., \& Summers, R. (Eds.). (1991). Interpreting Statutes. A Comparative Study.

Dartmouth, England: Aldershot.

Marmor, A. (2001). Interpretación y teoría del Derecho (M. Medina Hurtado, Trans.). Barcelona, Spain: Gedisa.

Moreso, J.J. (1997). La indeterminación del Derecho y la interpretación de la constitución. Madrid, Spain: Centro de Estudios Políticos y Constitucionales.

Nino, C.S. (1994). Derecho, moral y política. Barcelona, Spain: Ariel.

Raz, J. (1979). The Rule of Law and its Virtue. In The Authority of Law: Essays on law and morality (pp. 210-229). Oxford, England: Clarendon Press.

Raz, J. (1994). The Politics of the Rule of Law. In Ethics in the Public Domain, Essays on the Morality of Law and Politics (pp. 370-378). Oxford, England: Clarendon Press. 
Raz, J. (1996). Why interpret? Ratio Iuris, 9(4), 349-363.

Rodríguez-Toubes Muñiz, J. (2014). Aspectos de la interpretación jurídica (un mapa conceptual). Anuario de filosofía del Derecho, XXX, 339-369.

Ross, A. (1970). Sobre el Derecho y la justicia (G. Carrió, Trans.). Buenos Aires, Argentina: Eudeba. von Savigny, F.K. (1878). Sistema del Derecho Romano actual (J. Mesía \& M. Poley, Trans.). Madrid, Spain: Centro editorial de Góngora. (Original work published 1840).

Summers, R.S. (2006). Form and Function in a Legal System. A General Study. New York, NY: Cambridge University Press.

Tarello, G. (1971). Orientamenti analitico-linguistici e teoria dell'interpretazione giuridica. Rivista trimestrale di diritto e procedura civile, XXV, 1-18.

Tarello, G. (1980). L'interpretazione della legge. Milan, Italy: Giuffrè.

Wendel, W.B. (2005). Professionalism as Interpretation (Cornell Law Faculty Publications Paper 27). Retrieved from: http://scholarship.law.cornell.edu/lsrp_papers/27.

Wróblewski, J. (1985). Constitución y teoría general de la interpretación jurídica (A. Azurza, Trans.). Madrid, Spain: Civitas.

Wróblewski, J. (1992a). Legal Language and Interpretation. In A. Aarnio \& N. MacCormick (Eds.), Legal Reasoning (vol. II, The International Library of Essays in Law and Legal Theory) (pp. 401-417). Dartmouth, England: Aldershot.

Wróblewski, J. (1992b). The Judicial Application of Law. Dordrecht, The Netherlands: Kluwer Academic Publisher.

\section{NOTAS}

1. Ver por todos, Lifante Vidal 2018: esp. 19-28.

2. Esta distinción de Gianformaggio entre la interpretación noética y la dianoética coincide en gran medida con distinciones similares de otros autores. Así por ejemplo, como acabamos de ver, Wróblewski 1985: 22 distingue dos situaciones de comunicación: la comprensión directa de un lenguaje y la existencia de dudas que deben ser superadas precisamente por la interpretación (en lo que él considera como su sentido estricto). Para una presentación de la polémica en la doctrina polaca entre esta concepción más estricta de la interpretación y una concepción más amplia, según la cual la interpretación sería una actividad omnipresente, puede verse Grabowski 2015. También Marmor (siguiendo en este punto las aportaciones de Wittgenstein) distingue entre “cuestiones de significado" y “cuestiones de interpretación” (Marmor 2001: 36ss.). Las cuestiones de significado -dice Marmor- están gobernadas por reglas (semánticas), mientras que esto no ocurre en el caso de la interpretación, donde nos encontraríamos con paradigmas, pero no con reglas. La "interpretación" se configuraría entonces, para este autor, como una excepción al entendimiento o comprensión inmediata.

3. Cfr. Wróblewski 1985: 21ss. y Wróblewski 1992b: 87ss. Pero para Wróblewski este segundo sentido de "interpretación" todavía lo sería en un sentido amplio, y ello porque este autor introduce en su análisis un tercer sentido de interpretación, a la que denomina "interpretación sensu stricto", entendiendo por tal "la determinación de un significado de una expresión lingüística cuando existen dudas referentes a ese significado en un caso concreto de comunicación". El tipo de objeto a interpretar sería en este tercer caso -para Wróblewski- el mismo que en el caso de la interpretación sensu largo: entidades lingüísticas; pero se requiere 
además que nos encontremos ante la presencia de dudas sobre su significado, siendo dichas dudas las que generan la necesidad de llevar a cabo una actividad interpretativa en este sentido estricto. Wróblewski distingue así dos situaciones de comunicación: la comprensión directa de un lenguaje y la existencia de dudas que han de ser superadas precisamente por esta interpretación sensu stricto. Pero si nos fijamos, lo que diferenciaría a ambos casos sería, entonces la distinta actividad que se requiere para "captar" o "establecer" el significado de la expresión lingüística en cada una de estas situaciones (de lo que nos acabamos de ocupar) y no tanto el diferente tipo de objeto que se interpreta.

4. Sobre esta evolución histórica, puede verse Bobbio 1938: 7ss.

5. Estos dos sentidos creo que vendrían a coincidir con la contraposición que Guastini (véase, por ejemplo, Guastini 2012) realiza entre "interpretación jurídica" (para referirse a lo que aquí llamamos "interpretatio legis") y la "construcción jurídica" (que se correspondería con todas las actividades que se incluyen en la "interpretatio iuris"). Sobre esta distinción de Guastini me he ocupado con detalle en un trabajo anterior (Lifante Vidal 2018: 110-114).

6. Este trabajo compara los argumentos usados para interpretar leyes, pero un poco después el mismo grupo de trabajo llevó a cabo otra comparación respecto a la interpretación de los precedentes (MacCormick \& Summers 1997).

7. En un sentido similar, Ross sostiene que la caracterización clásica que suele hacerse de la distinción entre la interpretación "objetiva" y la "subjetiva", según la cual ambos tipos interpretativos se diferenciarían por el objetivo que cada una de ellas persigue (en un caso, descubrir el significado del texto y, en el otro, la intención del autor), resulta insostenible. Frente a este planteamiento, considera que la intención del autor de la expresión es un fenómeno de conciencia interno y, por tanto, inaccesible; en este sentido lo que se entiende por interpretación subjetiva coincidiría con aquella interpretación en la cual se toman en cuenta, además de la expresión lingüística, otros factores relevantes como son el contexto y la situación (Ross 1970: 117ss.).

8. Debemos ser conscientes de que interpretaciones incompatibles pueden ser apoyadas incluso por argumentos de la misma categoría: dos argumentos lingüísticos (o sistemáticos...) pueden apoyar diferentes interpretaciones. Cfr. MacCormick 1993.

9. No es fácil encontrar ejemplos de teorías puramente literalistas de la interpretación jurídica, pues muchos de los problemas de interpretación se producen precisamente por las indeterminaciones lingüísticas. Para una buena defensa de la interpretación literal en el ámbito jurídico, puede verse Laporta 2007: cap. VIII.

10. Aunque en ocasiones el subjetivismo (o intencionalismo) es considerado equivalente al originalismo interpretativo, Rodríguez-Toubes Muñiz muestra el error que ello conlleva. Este autor realiza una doble distinción entre, por un lado, concepciones objetivistas y subjetivistas; y, por otro lado, concepciones originalistas y evolutivas. Mientras que la primera distinción haría referencia a si el significado atribuible es el significado "que se desprende del texto" o el significado que quiso darle su autor; la segunda distinción haría referencia a si el significado (bien sea el atribuible objetivamente al texto o bien subjetivamente al legislador) es el que tenía en el momento en que se promulgó la norma, o en el momento de su interpretación (y en su caso aplicación). Aunque normalmente se suelen asociar subjetivismo y originalismo, el autor señala que no deben confundirse pues no siempre van de la mano. Y así, por ejemplo, señala que Scalia sería al mismo tiempo representante de una concepción objetivista y originalista de la interpretación (Rodríguez-Toubes Muñiz 2014: 324).

11. Cfr. Hart 1983. Moreso 1997 acuñó el rótulo de "teoría de la vigilia" para referirse precisamente a una teoría intermedia entre la "pesadilla" del escepticismo, y el "noble sueño" del formalismo.

12. Cfr. Guastini 2008: 51ss. Este autor ha sostenido en diversas ocasiones que, dado que todos los casos son en realidad difíciles (pues la indeterminación del Derecho es para él omnipresente), la 
única respuesta plausible sería el escepticismo. Sin embargo, en escritos posteriores (Guastini 2014) parece aceptar que puede haber casos -aunque pocos- en los que no haya varias posibilidades interpretativas, sino sólo una.

13. Como hemos visto antes, esta distinción de dos usos de los enunciados interpretativos es señalada por Guastini 1999: 205).

14. En mi opinión, una buena teoría de la argumentación jurídica debe servir no sólo para describir o analizar las argumentaciones reales, sino también para evaluarlas. En esto sigo a Atienza 2013.

15. Sigo aquí muy de cerca a Atienza 2017: cap I.

16. Cfr. Dworkin 2007: 11ss. y 243ss.

17. Aunque, obviamente, la plausibilidad de esta combinación dependerá de la concepción de las normas que se sostenga.

18. Uso la expresión "práctica social" en el mismo sentido que, por ejemplo, lo hace Dworkin 1986.

19. Por supuesto, con la expresión "Estado constitucional" no me estoy refiriendo a cualquier Estado que tenga una constitución formal (una mera norma jerárquicamente superior al resto), sino a los Estados con una constitución en un sentido material, que sería característica del llamado constitucionalismo político. Cfr. Aguiló Regla 2019.

20. Podría alegar que la "razón" para esa regularidad es simplemente una cuestión histórica, pero esta respuesta se refiere, como mucho, a las causas o razones explicativas de esa regularidad, no a las razones justificativas: tendría que admitir que la situación podría ser completamente diferente.

21. Cfr. Atienza 2013: 110.

22. En este punto me distancio de la tesis de Guastini, para quien la interpretación jurídica se adscribe necesariamente al género de las interpretaciones de textos y no tendría nada en común con otros géneros interpretativos (por ejemplo, el de prácticas sociales). Sobre ello véase Lifante Vidal 2018: 117ss.

23. En un artículo titulado precisamente “¿Por qué interpretar?”, Joseph Raz se pregunta por qué la interpretación es una actividad central en las prácticas jurídicas. Él considera que la respuesta a esta preguntase encuentra en el estatus de las fuentes del Derecho. Es por esta razón por la que considera que la "continuidad" y la "autoridad" ofrecen la clave para esta cuestión, pero la cuestión -distinta- respecto a cómo interpretar el Derecho se conecta, en su opinión, con otros factores, en particular con la "equidad" y el "desarrollo jurídico" (Raz 1996: 349ss.).

24. En este sentido, Dworkin (2014: 171ss.) distingue diversos tipos de interpretación, dependiendo de las "ocasiones" en que se llevan a cabo, cada una de las cuales se definiría no tanto por el objeto a interpretar, sino precisamente por la finalidad perseguida, y también en parte por el tipo de relación que existe en cada caso entre el intérprete y el objeto a interpretar. Estos tipos $\mathrm{u}$ ocasiones interpretativas son la colaborativa, la explicativa y la conceptual. La interpretación colaborativa sería aquella en la que se asume que el objeto de la interpretación tiene un autor, el cual ha iniciado un proyecto (una empresa) en el que el intérprete también se implica e intenta avanzar. De este modo, autor e intérprete compartirían el propósito general perseguido por el proyecto. La interpretación explicativa no presupone que los intérpretes y los creadores del objeto o evento a interpretar estén en el mismo nivel (no comparten un proyecto). En la interpretación conceptual el intérprete busca el significado de un concepto que ha sido creado no por autores individuales, sino por la propia comunidad; comunidad que incluye al propio intérprete, de modo que en esta ocasión interpretativa la distinción entre creador del objeto a interpretar e intérprete se desvanece hasta cierto punto. La interpretación jurídica (fundamentalmente la interpretación judicial) es para Dworkin un supuesto de interpretación colaborativa. 
25. Incluyendo también la de autores escépticos como Guastini o Kelsen. Es sabido que este último autor distingue entre la interpretación auténtica y la no auténtica; pero dentro de esta última se ocupa exclusivamente de caracterizar la interpretación que lleva a cabo la ciencia del Derecho (cfr. Kelsen 1986: 349ss).

26. Esta distinción entre interpretación en abstracto y en concreto ha sido trazada por muy diversos autores, con terminologías en ocasiones distantes. Entre otros, pueden verse: Ross 1970: 113, quien distingue entre interpretación por significado e interpretación por resultado; MacCormick 1978: 94ss., este autor habla de problemas de interpretación y problemas de calificación; Wróblewski 1992a: 405-408; y Guastini (por todos, 1999).

27. Me he ocupado recientemente de la caracterización de estas concepciones escépticas de la interpretación jurídica (véase, Lifante Vidal 2018: cap. II).

28. Dicho en términos dworkinianos, podríamos decir que se trataría de un supuesto de interpretación colaborativa.

29. Como señaló Hart, ocupar un lugar o rol determinado en el marco de una organización social (participar en un proyecto) implica ser responsable de las realización de esos deberes o de hacer lo necesario para satisfacerlos (Hart 1992: 212). Éste es precisamente uno de los significados del término "responsabilidad" que Hart distingue, en particular el de "responsabilidad de rol".

30. En este mismo sentido, Wendel llama la atención sobre el carácter autodestructivo e irracional que tendría para los abogados no preocuparse por la mejora de la práctica jurídica, dado que se trata de una práctica necesaria para poder defender los propios intereses particulares: "cualquier argumento ético plausible, es decir, digno del respeto de otros situados en la misma situación, debe tener en cuenta las consecuencias de la manipulación generalizada de las normas jurídicas formales" (Wendel 2005: 1210).

31. Para una caracterización de este concepto formal de "rule of law" y su distinción del concepto sustantivo, puede verse Craig 1997 y Barber 2004. Esta terminología es criticada por Gardner 2012, quien duda de la adecuación de estas etiquetas para referirse a los rasgos incluidos en el ideal de "rule of law", al menos tal y como es desarrollado por Fuller 1969 o Raz 1979 y Raz 1994.

32. También Raz ha sostenido, en un sentido similar, que la "observance of the rule of law is necessary if the law is to respect human dignity" (Raz 1979: 221). Respetar la dignidad humana implica tratar a los seres humanos como personas capaces de planificar y trazar su futuro. De este modo, respetar la dignidad de las personas implica respetar su autonomía, su derecho a controlar su futuro.

33. Cfr. Atiyah \& Summers 1987: 11ss.). Para estos autores, la formalidad (en cualquiera de esos sentidos) es una cuestión de grado, de modo que una razón puede ser más o menos formal y/o sustancial. Un razonamiento jurídico sería "formalista" cuando se produce una degeneración de uno o más de los aspectos formales presentes en el Derecho en detrimento de sus aspectos sustantivos. En realidad, para estos autores, serían rechazables tanto el exceso de formalidad como el exceso de sustantividad. En un trabajo posterior en solitario, Summers 2006 se ha apartado en cierto modo de esta contraposición formal/sustancial optando por una definición más amplia de lo que implica la "forma" en el Derecho, pero seguiría estando de acuerdo con los riesgos del exceso de "formalismo".

34. Me he ocupado de desarrollar esta concepción de la seguridad y previsibilidad jurídica en un trabajo anterior (Lifante Vidal 2013), donde junto a esa dimensión objetiva y subjetiva, analizaba también la dimensión temporal de la previsibilidad.

35. Tomo la cita de Fuller 1934: 432.

36. En un sentido parecido se pronuncia MacCormick, quien sostiene que la interpretación jurídica exigirá reflexionar sobre los "valores y principios adecuados a las instituciones de las sociedades, los estados y las comunidades internacionales y supranacionales en las que habitamos. Necesitamos pensar sobre el significado del constitucionalismo [...] la democracia, el principio de legalidad, la separación de poderes, la justicia procedimental, la equidad, los 
derechos humanos y la integridad de las instituciones estatales" (MacCormick 2010: 77). También Dworkin 2010 considera que el jurista (aunque él está pensando casi exclusivamente en el juez), en su tarea interpretativa, acaba desempeñando necesariamente funciones de filósofo moral y político.

\section{RESÚMENES}

Este trabajo pretende defender una concepción constructivista o dependiente de valores de la interpretación jurídica y mostrar su superioridad frente a concepciones rivales. La interpretación jurídica es una actividad que implica la participación en la práctica del Derecho y se encuentra por tanto comprometida con los fines y valores que dotan de sentido a la práctica o, dicho de otro modo, con la mejora de la misma. En este sentido, el trabajo sostiene que para contestar a la pregunta sobre cómo debe interpretarse el Derecho debemos plantearnos previamente qué es lo que hace que el Derecho sea valioso; la evaluación de una determinada interpretación como mejor o peor que otras interpretaciones posibles dependerá precisamente de cómo aquélla contribuya a maximizar esos principios que hacen que consideremos valioso el Derecho. De este modo, la argumentación jurídica en el ámbito interpretativo tiene un componente moral y político, aunque ello no tiene por qué implicar desconocer sus peculiaridades, en especial, la relevancia de los elementos autoritativos.

This work aims at articulating a constructivist or value-dependent conception of legal interpretation and at arguing in defence of its superiority against rival conceptions. Legal interpretation is an activity that implies participating in legal praxis. Thus, it is committed to those ends and values which give meaning to it, or in other words, it is committed to improve such praxis. Accordingly, to answer the question of how to interpret law, we must first answer the question of what makes law valuable. To assess whether certain interpretation is better or worse than other alternative ones depends precisely on how it contributes to maximizing those principles making us consider law valuable. Legal argumentation for the purposes of legal interpretation has necessarily a moral and a political component; nonetheless, this does not imply disregarding the peculiarities of legal argumentation, particularly the relevance of authoritative elements.

\section{ÍNDICE}

Palabras claves: Interpretación jurídica, argumentación jurídica, escepticismo, cognoscitivismo, constructivismo

Keywords: Legal interpretation, legal argumentation, scepticism, cognitivism, constructivism

\section{AUTOR}

\section{ISABEL LIFANTE VIDAL}

Profesora titular de Filosofía del Derecho en la Universidad de Alicante 
Address: Facultad de derecho, Universidad de Alicante - Carretera San Vicente del Raspeig s/n 03690 San Vicente del Raspeig, Alicante - Spain

E-mail: isabel.lifante@ua.es 